Article

\title{
Cobalt and Nitrogen Co-Doped Graphene-Carbon Nanotube Aerogel as an Efficient Bifunctional Electrocatalyst for Oxygen Reduction and Evolution Reactions
}

\author{
Xiaochang Qiao ${ }^{1,2}$, Jutao Jin ${ }^{2}$, Hongbo Fan ${ }^{2, *}$, Lifeng Cui ${ }^{2}$, Shan $\mathrm{Ji}^{3}$, Yingwei $\mathrm{Li}^{1}$ \\ and Shijun Liao ${ }^{1, *}$ \\ 1 The Key Laboratory of Fuel Cell Technology of Guangdong Province, School of Chemistry and Chemical \\ Engineering, South China University of Technology, Guangzhou 510641, China; qiaoxc@dgut.edu.cn (X.Q.); \\ liyw@scut.edu.cn (Y.L.) \\ 2 School of Environment and Civil Engineering, Dongguan University of Technology, Dongguan 523808, \\ China; 2016809@dgut.edu.cn (J.J.); lifeng.cui@gmail.com (L.C.) \\ 3 College of Biological, Chemical Science and Chemical Engineering, Jiaxing University, Jiaxing 314001, China; \\ jishan@mail.zjxu.edu.cn \\ * $\quad$ Correspondence: fhb@dgut.edu.cn (H.F.); chsjliao@scut.edu.cn (S.L.); Tel.: +86-20-8711-3586 (S.L.)
}

Received: 13 June 2018; Accepted: 3 July 2018; Published: 7 July 2018

\begin{abstract}
In this study, a low-cost and environmentally friendly method is developed to synthesize cobalt and nitrogen co-doped graphene-carbon nanotube aerogel (Co-N-GCA) as a bifunctional electrocatalyst for the oxygen reduction reaction (ORR) and the oxygen evolution reaction (OER). The as-prepared Co-N-GCA has a hierarchical meso- and macroporous structure with a high $\mathrm{N}$ doping level (8.92 at. \%) and a large specific surface area $\left(456 \mathrm{~m}^{2} \mathrm{~g}^{-1}\right)$. In an alkaline medium, the catalyst exhibits superior ORR electrocatalytic activity with an onset potential $15 \mathrm{mV}$ more positive than $\mathrm{Pt} / \mathrm{C}$, and its diffusion-limiting current density is $29 \%$ higher than that of commercial $\mathrm{Pt} / \mathrm{C}$. The obtained Co-N-GCA is also highly active toward the OER, with a small overpotential of $408 \mathrm{mV}$ at a current density of $10 \mathrm{~mA} \mathrm{~cm}^{-2}$. Its overall oxygen electrode activity parameter $(\Delta \mathrm{E})$ is $0.821 \mathrm{~V}$, which is comparable to most of the best nonprecious-metal catalysts reported previously. Furthermore, Co-N-GCA demonstrates superior durability in both the ORR and the OER, making it a promising noble-metal-free bifunctional catalyst in practical applications for energy conversion and storage.
\end{abstract}

Keywords: graphene-carbon nanotube aerogel; cobalt and nitrogen co-doped; oxygen reduction reaction; oxygen evolution reaction

\section{Introduction}

Global energy and environmental issues have stimulated tremendous ongoing research into developing sustainable and environmentally friendly energy conversion and storage systems [1]. Creating highly active electrocatalysts for the oxygen reduction reaction (ORR) and the oxygen evolution reaction (OER) is crucial for the practical application of fuel cells, metal-air batteries, and electrolyzers. Currently, Pt-based catalysts are regarded as the most active ORR catalysts but are poor for the OER [2,3], while $\mathrm{IrO}_{2}$ and $\mathrm{RuO}_{2}$ are the most efficient catalysts for the OER but are poor for the ORR [4]. However, their commercialization has been greatly hindered by the scarcity of their materials, their consequent high cost, and their poor durability. As a result, extensive research efforts have been devoted to searching for low-cost alternatives with comparable catalytic activity to 
noble metal-based catalysts [5,6]. Due to the sluggish kinetics of the ORR and OER [7-9], it is highly challenging but imperative to develop a cheap and effective bifunctional electrocatalyst for the ORR and OER.

Extensive efforts have been made to develop non-noble-metal bifunctional electrocatalysts, such as transition metal oxides/sulfides [10-15], carbon-based materials [9,16-19], and perovskites [20]. Among these promising bifunctional ORR/OER catalysts, metal-nitrogen-doped carbon materials have attracted much attention due to their remarkable activity, tunable surface chemistry, fast electron transfer capacity, and economic viability [21-23]. However, substantially increasing the active sites of metal-nitrogen-doped carbon materials remains a great challenge. Recently, it was found that using nanosized carbon materials as supports, such as one-dimensional carbon nanotubes (CNTs) and two-dimensional graphene, can further improve electrocatalytic performance [24-27]. However, methods for the synthesis of metal-nitrogen-doped graphene/CNTs often requires the high temperature $\left(600-1200{ }^{\circ} \mathrm{C}\right)$ which limits the practical application. And both CNTs and graphene nanosheets are inclined to aggregate with each other due to strong Van der Waals interactions, and this greatly hinders the full utilization of the active sites for catalytic reactions [21,28,29].

Because of their three-dimensional network structure, extremely low density, high porosity, and large specific surface area, carbon-based aerogels have emerged as promising nanomaterials and are providing fascinating options for preparing new functional electrode materials [30]. The incorporation of CNTs into graphene to produce a graphene-CNTs hybrid aerogel not only could favor the dispersion of graphene and CNTs while maintaining the original properties of both graphene and CNTs, but also could yield a 3D, interconnected, conductive network structure. This aerogel network structure, for ORR, favors the $\mathrm{O}_{2}$ transformation to the active sites; for OER, facilitates timely transfer evolved $\mathrm{O}_{2}$ molecules. It is expected that a graphene-carbon nanotube aerogel could result in better electrocatalytic performance. However, to the best of our knowledge, there is no report about $\mathrm{M}-\mathrm{N}-\mathrm{C}$ graphene-CNTs aerogel as an efficient oxygen electrode catalyst.

Herein, we report a one-pot hydrothermal method to prepare a cobalt and nitrogen co-doped graphene-CNTs aerogel (Co-N-GCA) as a bifunctional electrocatalyst for the ORR and OER. Benefiting from abundant catalytic active sites and a unique hierarchical structure that increases the exposure of active sites and favors electron transfer and the diffusion of $\mathrm{O}_{2}$ molecules, the obtained electrocatalyst exhibits superior electrocatalytic activity towards both the ORR and the OER in an alkaline medium.

\section{Results and Discussions}

The inset in Figure 1a is a photo of the as-prepared Co-N-GCA aerogel after freeze drying, showing a black, low-weight, sponge-like material. The SEM images (Figure 1a,b) reveal that the Co-N-GCA exhibits a well-defined and interconnected 3D porous network structure. The CNTs are randomly and uniformly distributed between the graphene sheets. The pore walls consist of thin layers of a network, which are cross-linked with graphene sheets and CNTs. TEM images (Figure 1c,d) further confirm that the CNTs adhere tightly on the graphene substrates. No obvious CNT bundles or graphene agglomerates are observable. Few scattered metal nanoparticles could also be found by TEM inspection. The high-resolution TEM image (Figure S1) reveals the lattice fringe space of $0.47 \mathrm{~nm}$ is consistent with the (111) plane of cubic $\mathrm{Co}_{3} \mathrm{O}_{4}$ spinel-phase. Photos of the prepared Co-GCA and N-GCA aerogels and the corresponding SEM images are also provided in Figure S2.

The $\mathrm{N}_{2}$ adsorption-desorption isotherms and corresponding pore-size distribution curve of Co-N-GCA are shown in Figure 2. According to the IUPAC classification, the $\mathrm{N}_{2}$ adsorption-desorption isotherms are of type IV, with the amount of absorbed $\mathrm{N}_{2}$ monotonically increasing/decreasing at high relative pressure $\left(P / P_{0}>0.9\right)$; this is typically associated with capillary condensation, indicating the existence of mesopores $(2-50 \mathrm{~nm})$. Notably, the capillary condensation phenomenon occurs at a high relative pressure, indicating a large pore-size distribution. The hysteresis loop resembles type $\mathrm{H}_{3}$, suggesting open slit-shaped capillaries between the parallel layers of graphene. The surface area of Co-N-GCA was calculated to be $456 \mathrm{~m}^{2} \mathrm{~g}^{-1}$. The Barrett-Joyner-Halenda (BJH) method indicated that 
a hierarchical, meso- and macroporous material with a pore volume of $1.64 \mathrm{~cm}^{3} \mathrm{~g}^{-1}$ was formed by this method. This result is consistent with the SEM observations. The high surface area and hierarchically porous structure of Co-N-GCA would provide plenty of active sites and favor the mass transport of reactants and products, resulting in enhanced ORR/OER electrocatalytic activity.

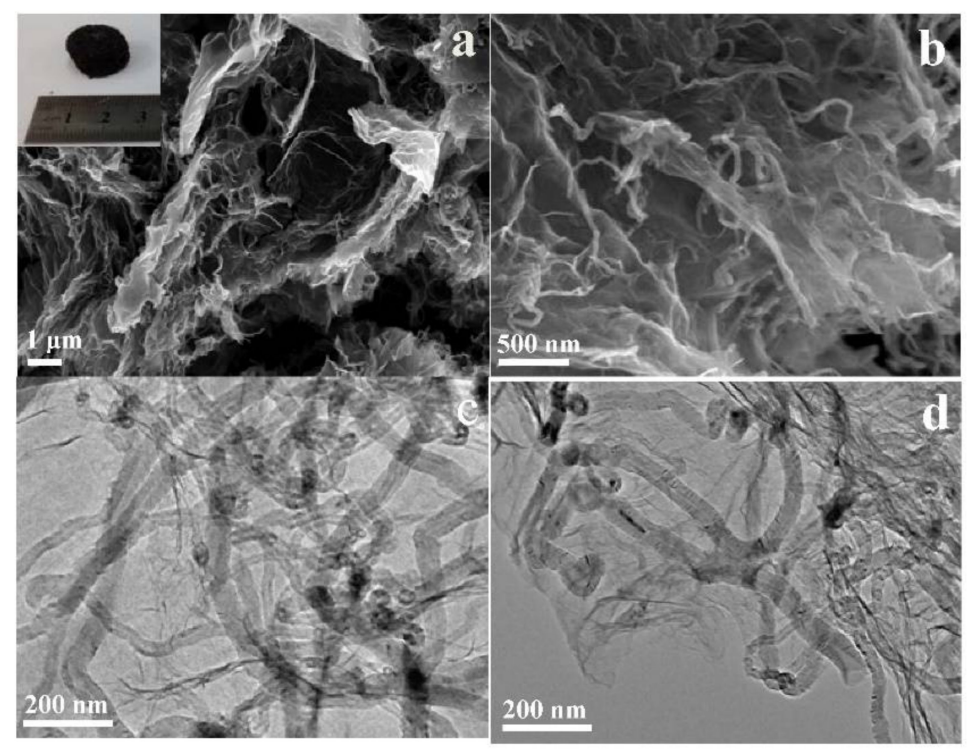

Figure 1. SEM (a,b) and TEM (c,d) images of Co-N-GCA (inset in (a) shows a digital photograph of the prepared aerogel).
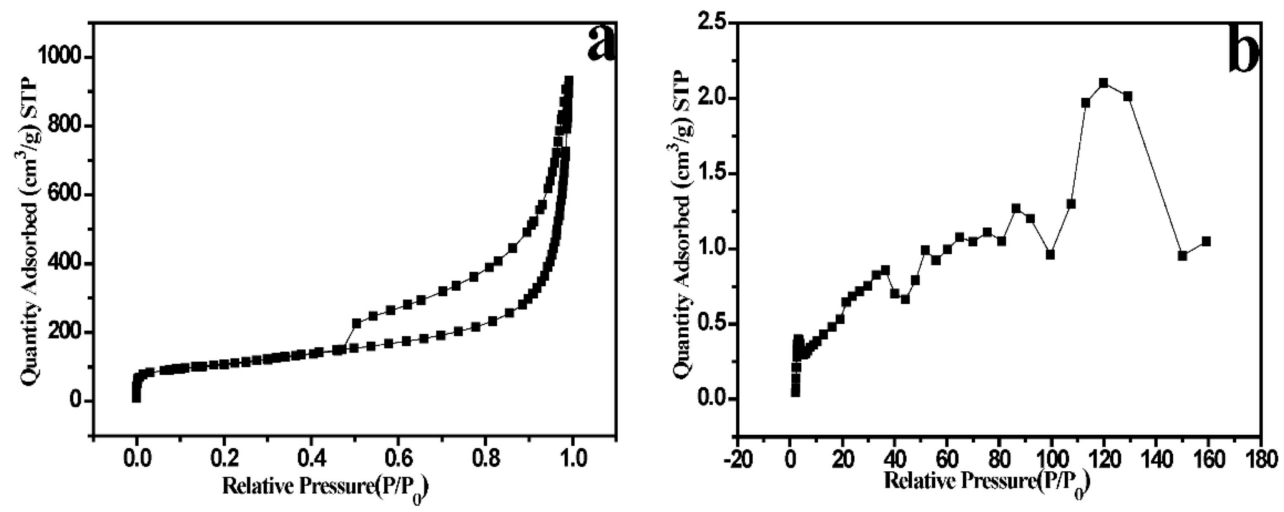

Figure 2. Nitrogen adsorption-desorption isotherms (a) and the corresponding pore-size distribution curve (b) of Co-N-GCA.

The elemental compositions of Co-N-GCA and N-GCA were investigated by X-ray photoelectron spectroscopy (XPS) analysis. As shown in Figure S2, the XPS survey spectrum of Co-N-GCA exhibited the signals of a C 1s peak $(\sim 284.5 \mathrm{eV})$, a N 1s peak $(\sim 398.1 \mathrm{eV})$, an O 1s peak $(\sim 531.1 \mathrm{eV})$, and a Co $2 \mathrm{p}$ peak $(\sim 780.1 \mathrm{eV})[22]$. The XPS results confirmed that $\mathrm{Co}$ and $\mathrm{N}$ elements were successfully doped into the carbon matrix, and the amounts of doped $\mathrm{Co}$ and N in Co-N-GCA were 0.44 and 8.92 at. \%, respectively. In the case of N-GCA, the doped N content was 8.68 at. \%, which was similar to that of Co-N-GCA (Table S1). XPS analysis shows that a high amount of doped $\mathrm{N}$ can be achieved by this method.

The deconvoluted high-resolution $\mathrm{N}$ 1s spectrum (Figure 3a) revealed four types of $\mathrm{N}$ species in Co-N-GCA: pyridinic N/Co-N $\mathrm{N}_{\mathrm{x}}(398.9 \mathrm{eV})$, pyrrolic $\mathrm{N}(400.0 \mathrm{eV})$, graphitic $\mathrm{N}(401.2 \mathrm{eV})$, and oxidated $\mathrm{N}(405.1 \mathrm{eV})$ [31]. Their corresponding contents are $47.5,34.8,13.5$, and 4.2 at. \%, respectively (Table S2) [32]. Notably, most of the doped $\mathrm{N}$ is pyridinic ( 47.5 at. \%). It has been reported that the ORR 
active sites in $\mathrm{N}$-doped carbon materials are carbon atoms with Lewis basicity next to pyridinic $\mathrm{N}$ [33]. We expected the as-prepared Co-N-GCA to exhibit outstanding electrocatalytic performance due to the high amount of pyridinic $\mathrm{N}$. Meanwhile, $\mathrm{M}-\mathrm{N}_{\mathrm{x}}$ structure have also been reported to contribute to ORR/OER active sites apart from the $\mathrm{N}-\mathrm{C}$ active sites [34]. The high-resolution $\mathrm{N} 1$ s spectrum and distribution of each N species of N-GCA are presented in Figure S3 and Table S2, respectively.
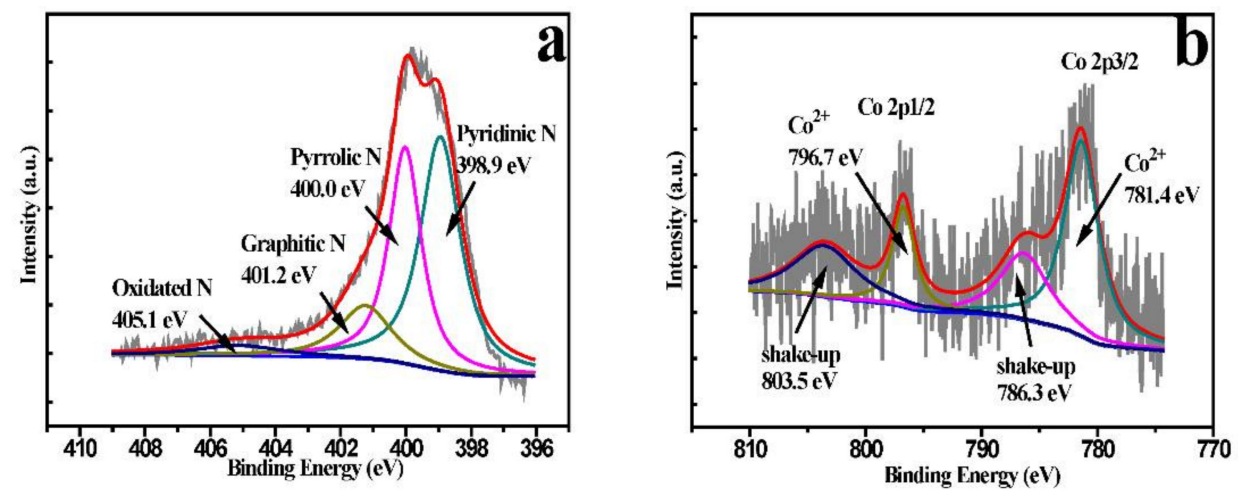

Figure 3. The X-ray photoelectron spectroscopy (XPS) high-resolution N 1s (a) and Co 2p (b) spectra of Co-N-GCA.

Figure $3 \mathrm{~b}$ presents a high-resolution XPS spectrum of Co $2 \mathrm{p}$. The peaks situated at $781.4 \mathrm{eV}$ and $796.7 \mathrm{eV}$ with two weak shake-up (satellite) peaks are assigned to the Co $2 \mathrm{p} 3 / 2$ and Co $2 \mathrm{p} 1 / 2$ atomic orbitals.

We have also performed inductively coupled plasma mass spectroscopic measurements to analyze the content of $\mathrm{Co}$, the result showed a relatively low cobalt content of $\sim 0.91 \mathrm{wt}$. \% in the Co-N-GCA sample. Meanwhile, we also performed element analysis test, the result showed the $\mathrm{N}$ content in the Co-N-GCA is 17.5 wt. \%.

Figure 4a presents the XRD patterns of Co-N-GCA, N-GCA, and GCA. All three samples show two broad, weak diffraction peaks at $2 \theta \approx 24^{\circ}$ and $43.7^{\circ}$, which correspond to the (002) and (100) reflections of the graphitic peak (PDF\#41-1487), respectively, confirming the graphitic crystal structure [35]. For Co-N-GCA, no metal peaks or other than carbon are observed, which may be due to the low Co content.
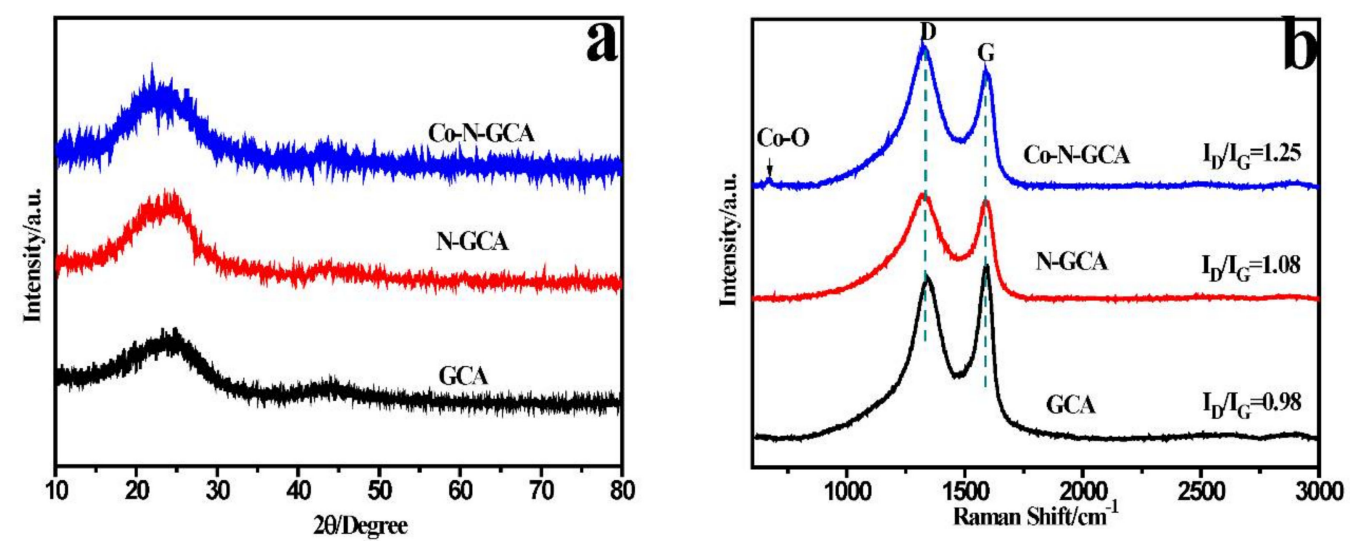

Figure 4. XRD patterns (a) and Raman spectra (b) of Co-N-GCA, N-GCA, and GCA.

Figure $4 \mathrm{~b}$ shows the Raman spectra of Co-N-GCA, N-GCA, and GCA. All of the samples exhibit two prominent peaks at ca. $1580 \mathrm{~cm}^{-1}$ and $1335 \mathrm{~cm}^{-1}$, corresponding to the characteristic D and G bands, respectively. The $\mathrm{D}$ band belongs to the breathing modes of $\mathrm{sp}^{2}$ atoms in rings, whereas the $\mathrm{G}$ band is assigned to stretching in all pairs of $\mathrm{sp}^{2}$ atoms in both rings and chains [36]. Generally, 
the intensity ratio of the $\mathrm{D}$ and $\mathrm{G}$ bands $\left(\mathrm{I}_{\mathrm{D}} / \mathrm{I}_{\mathrm{G}}\right)$ is used to quantify the extent of defects in carbon materials. The $\mathrm{I}_{\mathrm{D}} / \mathrm{I}_{\mathrm{G}}$ ratios for Co-N-GCA, N-GCA, and GCA were $1.25,1.08$, and 0.98 , respectively, indicating that Co-N-GCA had more structural defects. In the Raman spectra of Co-N-GCA, there is a small peak at $672 \mathrm{~cm}^{-1}$, which can be indexed to Co-O.

The electrocatalytic performance of Co-N-GCA for the ORR was initially evaluated separately by cyclic voltammetry (CV) in $\mathrm{N}_{2}$ - and $\mathrm{O}_{2}$-saturated $0.1 \mathrm{M} \mathrm{KOH}$ solutions. As shown in Figure $5 \mathrm{a}$, the CV curve of Co-N-GCA was virtually featureless in the $\mathrm{N}_{2}$-saturated electrolyte, while displayed well-defined oxygen reduction cathodic peaks in the $\mathrm{O}_{2}$-saturated electrolyte. Notably, Co-N-GCA had the more positive ORR peak potential ( $0.837 \mathrm{~V}$ vs. RHE) than commercial $20 \mathrm{wt}$. \% Pt/C $(0.841 \mathrm{~V}$ vs. RHE) (Figure S4), indicating the superior ORR catalytic activity of Co-N-GCA.
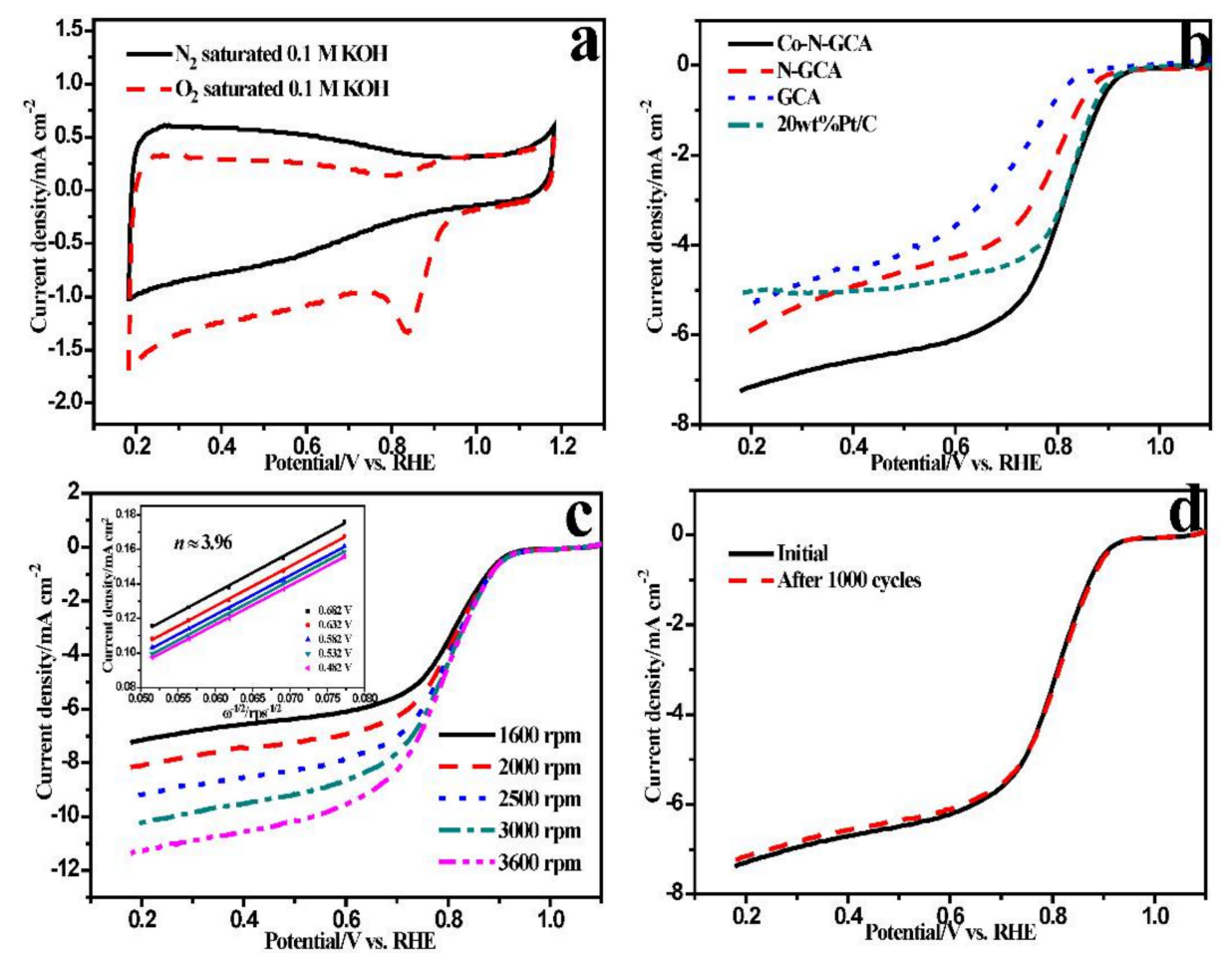

Figure 5. (a) Cyclic voltammetry (CV) curves of Co-N-GCA in $\mathrm{N}_{2}$ - and $\mathrm{O}_{2}$-saturated $0.1 \mathrm{M} \mathrm{KOH}$ solutions; (b) linear sweep voltammetry (LSV) curves of Co-N-GCA, N-GCA, GCA, and commercial 20 wt. \% Pt/C at a rotation speed of $1600 \mathrm{rpm}$; (c) LSV curves of Co-N-GCA at 1600-3600 rpm rotation speeds (inset shows the corresponding K-L plots); (d) LSV plots of Co-N-GCA at a rotation speed of $1600 \mathrm{rpm}$ before and after $1000 \mathrm{CV}\left(50 \mathrm{mV} \mathrm{s}^{-1}\right)$ cycles.

To further investigate the ORR activity, the linear sweep voltammetry (LSV) curve of Co-N-GCA was recorded at $1600 \mathrm{rpm}$ in $\mathrm{O}_{2}$-saturated $0.1 \mathrm{M} \mathrm{KOH}$ solution. For comparison, we present the LSV curves of Co-N-GCA, N-GCA, GCA, and commercial $20 \mathrm{wt}$ \% Pt/C in Figure $5 \mathrm{~b}$. In terms of the onset potentials and diffusion-limiting current densities of the ORR, Figure $5 b$ show that the ORR activity follows the order GCA $<$ N-GCA $<$ Co-N-GCA. The Co-N-GCA is more electrocatalytically active toward the ORR than $\mathrm{Pt} / \mathrm{C}$, with an onset potential of $0.975 \mathrm{~V}$ (vs. RHE), which is $15 \mathrm{mV}$ more positive than that of commercial Pt/C. The diffusion-limiting current density of Co-N-GCA (at $0.6 \mathrm{~V}$ ) is about $29 \%$ higher than that of commercial $\mathrm{Pt} / \mathrm{C}$, which further indicates that the mass transport on Co-N-GCA is more efficient than on $\mathrm{Pt} / \mathrm{C}$.

To gain more information on the ORR kinetics of the Co-N-GCA catalyst, LSV curves were recorded in an $\mathrm{O}_{2}$-saturated $0.1 \mathrm{M} \mathrm{KOH}$ solution at various rotation rates, increasing from 1600 to $3600 \mathrm{rpm}$ (Figure 5c). The diffusion current density of oxygen reduction increased with the rotation rate, owing to enhanced mass transport. In addition, the K-L plots at different electrode potentials 
displayed good linearity. The K-L equation was adopted to calculate the electron transfer number (n) of Co-N-GCA in the potential range from $0.482 \mathrm{~V}$ to $0.682 \mathrm{~V}$, and an average $\mathrm{n}$ value of 3.96 was obtained, indicating that the ORR proceeded via a four-electron pathway.

The stability of the Co-N-GCA catalyst was further evaluated by LSV in $\mathrm{O}_{2}$-saturated $0.1 \mathrm{M} \mathrm{KOH}$ at $1600 \mathrm{rpm}$ for 1000 continuous cycles. As shown in Figure 5d, there wasn't an obvious change in the half-wave potential of Co-N-GCA after 1000 continuous cycles, suggesting that Co-N-GCA has excellent ORR stability in an alkaline medium.

The electrocatalytic OER activity of Co-N-GCA was investigated by LSV measurements, which were conducted in $\mathrm{O}_{2}$-saturated $0.1 \mathrm{M} \mathrm{KOH}$ at $1600 \mathrm{rpm}$. For comparison, the OER with N-GCA, GCA, and $\mathrm{IrO}_{2} / \mathrm{C}$ was also performed under the same conditions (Figure 6a). OER catalytic activities are commonly judged by the potential required to oxidize water at a current density of $10 \mathrm{~mA} \mathrm{~cm}{ }^{-2}$. Significantly, compared with the standard reaction potential $(1.23 \mathrm{~V})$, the Co-N-GCA composite can reach $10 \mathrm{~mA} \mathrm{~cm}^{-2}$ with a small overpotential ( $\eta$ ) of $408 \mathrm{mV}$, while N-GCA, GCA, and $\mathrm{IrO}_{2} / \mathrm{C}$ reach the same current density with overpotentials $(\eta)$ of 515,685 , and $377 \mathrm{mV}$, respectively.
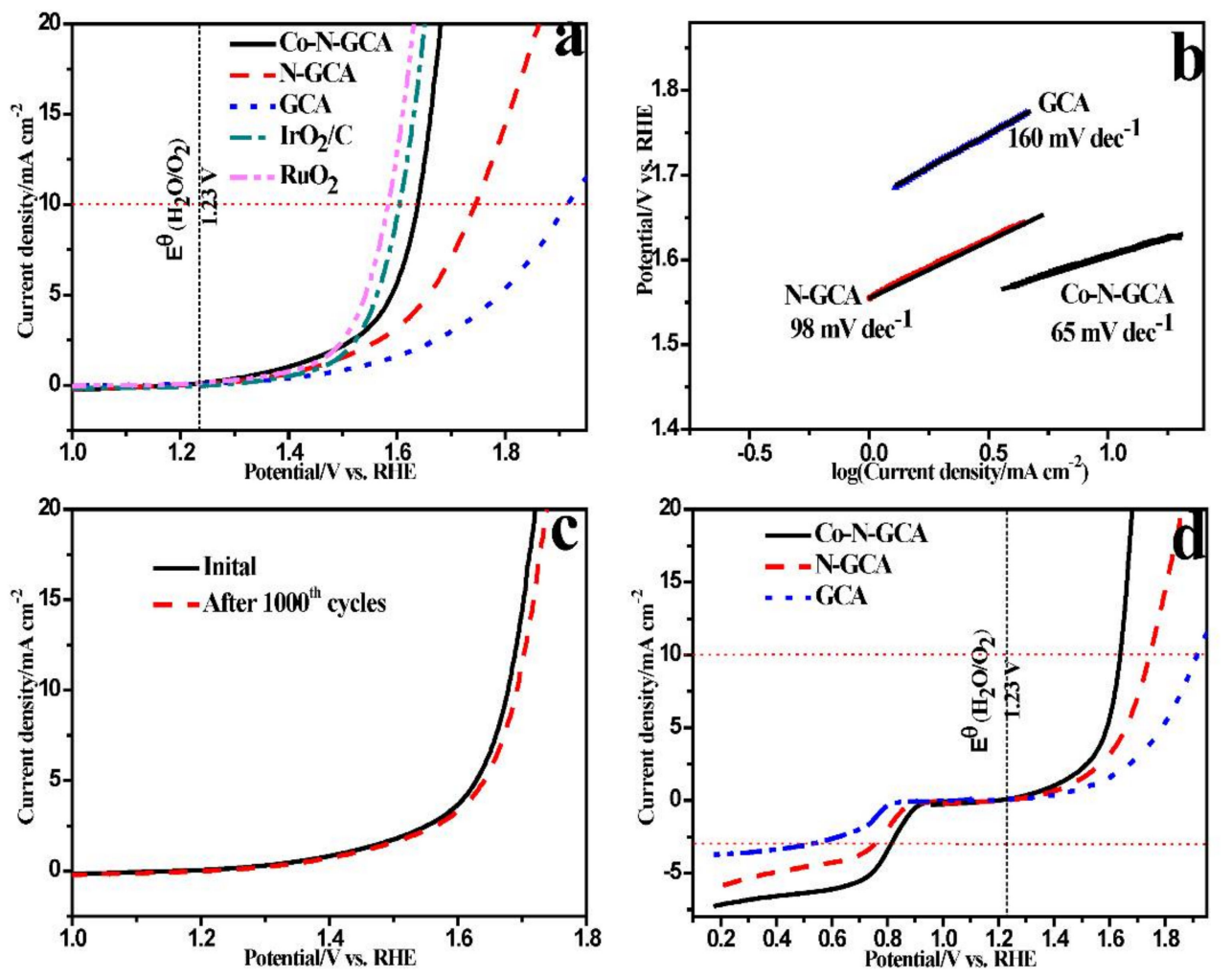

Figure 6. Oxygen evolution reaction (OER) LSV curves of Co-N-GCA, N-GCA, GCA, $\mathrm{IrO}_{2} / \mathrm{C}$, and $\mathrm{RuO}_{2}$ at $1600 \mathrm{rpm}$ in $\mathrm{O}_{2}$-saturated $0.1 \mathrm{M} \mathrm{KOH}$ solution (a); OER Tafel plots of Co-N-GCA, N-GCA, and GCA (b); OER LSV plots of Co-N-GCA in the beginning and after 1000 cycles (c); and the overall LSV curves of Co-N-GCA, N-GCA, and GCA at $1600 \mathrm{rpm}$ in $\mathrm{O}_{2}$-saturated $0.1 \mathrm{M} \mathrm{KOH}$ solution (d).

The Tafel plots were also generated to study the OER kinetics of these catalysts. As shown in Figure $6 \mathrm{~b}$, the resulting Tafel slopes of Co-N-GCA, N-GCA, and GCA were found to be $\sim 65, \sim 98$, and $\sim 160 \mathrm{mV} \mathrm{dec}^{-1}$, respectively. The Co-N-GCA composite exhibited the smallest Tafel slope, suggesting its extremely favorable reaction kinetics.

Accelerated stability tests in $\mathrm{O}_{2}$-saturated $0.1 \mathrm{M} \mathrm{KOH}$ at room temperature for $\mathrm{Co}-\mathrm{N}$-GCA were also carried out to investigate its durability for the OER. As shown in Figure 6c, after 1000 continuous potential cycles, the overpotential of Co-N-GCA increased by only $20 \mathrm{mV}$ at a current density of $10 \mathrm{~mA} \mathrm{~cm}^{-2}$, indicating Co-N-GCA has superior electrocatalytic stability for the OER. 
The overall electrocatalytic activity of a bifunctional electrocatalyst as an oxygen electrode can be evaluated by taking the difference in potential between the OER current density at $10 \mathrm{~mA} \mathrm{~cm}^{-2}$ and the ORR current density at $-3 \mathrm{~mA} \mathrm{~cm}^{-2}(\Delta \mathrm{E})$. If the difference $\Delta \mathrm{E}$ is smaller, this usually indicates the material is more suitable for practical applications. The overall electrocatalytic activities for the above catalysts are shown in Figure $6 \mathrm{~d}$. The $\Delta \mathrm{E}$ values for Co-N-GCA, N-GCA, and GCA are $0.821,0.983$, and $1.389 \mathrm{~V}$, respectively, showing that the bifunctional catalytic activities of the samples followed the order Co-N-GCA > N-GCA > GCA. More importantly, the $\triangle \mathrm{E}$ value for Co-N-GCA is comparable to or even much smaller than many nonprecious metal-based bifunctional oxygen electrode catalysts reported previously (Table S3), suggesting the as-prepared Co-N-GCA is an effective bifunctional catalyst for the ORR and OER.

\section{Materials and Methods}

\subsection{Materials}

Graphite powder was purchased from Sigma Aldrich (Saint Louis, MO, USA). Multiwall carbon nanotubes were bought from Tsinghua University (Beijing, China). Urea and cobalt(II) acetate tetrahydrate were obtained from Sinopharm Chemical Reagent Co., Ltd. (Shanghai, China). Nafion (5 wt. \%) was obtained from DuPont (Wilmington, DE, USA). Commercial Pt/C (20 wt. \%) catalyst was purchased from Johnson Matthey (London, UK). $\mathrm{RuO}_{2}$ was obtained from Sigma-Aldrich (Saint Louis, $\mathrm{MO}$, USA). $\mathrm{IrO}_{2} / \mathrm{C}(20 \mathrm{wt}$. \%) catalyst was prepared by a method reported in the literature [37]. Carbon nanotubes were acid-treated with concentrated $\mathrm{HNO}_{3}$ at $110{ }^{\circ} \mathrm{C}$ for $3 \mathrm{~h}$ to remove metal impurities and enhance wettability. Other reagents were of analytical grade and were used as received without further purification. Graphite oxide (GO) was synthesized from natural graphite flakes by a modified Hummers method [38].

\subsection{Preparation of the Catalysts}

GO (40 mg) was dispersed in $40 \mathrm{~mL}$ deionized water. Acid-treated carbon nanotubes $(4 \mathrm{mg})$, urea $(6 \mathrm{~g})$, and cobalt(II) acetate tetrahydrate $(30 \mathrm{mg})$ were added to the GO solution. The obtained mixture was ultrasonically treated for $2 \mathrm{~h}$ to form a suspension. Subsequently, the stable suspension was transferred into a $50 \mathrm{~mL}$ Teflon-lined autoclave and heated at $170{ }^{\circ} \mathrm{C}$ for $10 \mathrm{~h}$. After the autoclave cooled to room temperature, the resulting hydrogel was washed with deionized water several times, followed by freeze drying. The resulting material is denoted as Co-N-GCA. For comparison, Co-GCA, $\mathrm{N}-\mathrm{GCA}$, and GCA were also prepared via similar procedures without the addition of urea, cobalt(II) acetate tetrahydrate, or both, respectively.

\subsection{Physical Characterization}

Scanning electron microscopy (SEM) images were collected on a Nova Nano 430 field emission scanning electron microscope (FEI, Hillsboro, OR, USA). Transmission electron microscopy (TEM) images were recorded on a JEM-2100HR transmission electron microscope (JEOL, Tokyo, Japan). Powder X-ray diffraction (XRD) patterns were obtained on a TD-3500 powder diffractometer (Tongda, Liaoning, China). Raman spectroscopy measurements were conducted on a Lab RAM Aramis Raman spectrometer (HORIBA Jobin Yvon, Edison, NJ, USA) with a laser wavelength of $632.8 \mathrm{~nm}$. Surface area and pore structure characteristics were analyzed by Brunauer-Emmett-Teller (BET) and Barrett-Joyner-Halenda (BJH) methods using the adsorption branch of the isotherms obtained on a Tristar II 3020 gas adsorption analyzer (Micromeritics, Norcross, GA, USA). X-ray photoelectron spectroscopy (XPS) measurements were carried out on an ESCALAB 250 X-ray photoelectron spectrometer (Thermo-VG Scientific, Waltham, MA, USA). 


\subsection{Electrochemical Measurements}

Electrochemical measurements were performed on an electrochemical workstation (Ivium, Eindhoven, the Netherlands) in a three-electrode configuration. An $\mathrm{Ag} / \mathrm{AgCl}$ electrode $(3 \mathrm{M} \mathrm{NaCl})$ and a Pt wire were used as the reference electrode and the counter electrode, respectively. $0.1 \mathrm{M}$ $\mathrm{KOH}$ solution was selected as the electrolyte. The catalyst ink was prepared by dispersing $5 \mathrm{mg}$ of the corresponding catalyst in $1 \mathrm{~mL}$ Nafion ethanol solution $(0.25 \mathrm{wt}$. \%), then dropping $20 \mu \mathrm{L}$ of the ink on a polished glassy carbon electrode with a diameter of $5 \mathrm{~mm}$, followed by drying under an infrared lamp.

Before the measurements, the electrolyte solution was purged with high-purity $\mathrm{N}_{2}$ or $\mathrm{O}_{2}$ gas for at least $30 \mathrm{~min}$. Cyclic voltammetry (CV) and linear sweep voltammetry (LSV) tests were performed with a sweep rate of $10 \mathrm{mV} \mathrm{s}^{-1}$. The $\mathrm{Ag} / \mathrm{AgCl}(3 \mathrm{M} \mathrm{NaCl})$ electrode was calibrated with respect to reversible hydrogen electrode (RHE), in $0.1 \mathrm{M} \mathrm{KOH}, \mathrm{E}(\mathrm{RHE})=\mathrm{E}(\mathrm{Ag} / \mathrm{AgCl})+0.982 \mathrm{~V}$.

For the ORR, the electron transfer number (n) per oxygen molecule was calculated with the Koutecky-Levich (K-L) equation as follows [11]:

$$
j^{-1}=j_{k}{ }^{-1}+\left(0.62 n F C D^{2 / 3} r^{-1 / 6} \omega^{1 / 2}\right)^{-1}
$$

where $\mathrm{j}$ and $\mathrm{j}_{\mathrm{k}}$ are the measured current density and the kinetic current density, respectively; $\mathrm{n}$ is the transferred electron number per oxygen molecule; $\mathrm{F}$ is the Faraday constant $\left(96,485 \mathrm{C} \mathrm{mol}^{-1}\right)$; $\mathrm{C}$ is the bulk concentration of $\mathrm{O}_{2}$ in $0.1 \mathrm{M} \mathrm{KOH}\left(1.2 \times 10^{-3} \mathrm{~mol} \mathrm{~L}^{-1}\right)$; $\mathrm{D}$ is the $\mathrm{O}_{2}$ diffusion coefficient of in 0.1 $\mathrm{M} \mathrm{KOH}\left(1.9 \times 10^{-5} \mathrm{~cm}^{2} \mathrm{~s}^{-1}\right) ; \gamma$ is the kinetic viscosity of the electrolyte $\left(0.01 \mathrm{~cm}^{2} \mathrm{~s}^{-1}\right)$; and $\omega$ is the angular velocity.

The Tafel plots were obtained by correcting the measured current density against the diffusion-limiting density to give the kinetic current density, calculated from:

$$
\mathrm{j}_{\text {kin }}=\frac{\mathrm{jj}_{\text {diff }}}{\mathrm{j}_{\text {diff }}-\mathrm{j}} \text {. }
$$

For the OER, the OER potential was IR corrected to compensate for the electrolyte's ohmic resistance by using the $\mathrm{E}-\mathrm{i} R$ relation, where $\mathrm{i}$ is the current and $\mathrm{R}$ is the uncompensated for ohmic electrolyte resistance $(\sim 45 \Omega)$ measured via high-frequency AC impedance.

\section{Conclusions}

In summary, a highly efficient bifunctional cobalt and nitrogen co-doped graphene-carbon nanotube aerogel (Co-N-GCA) has been designed and prepared using a simple one-pot hydrothermal method. By combining the merits of: (1) a synergistic effect between the abundant highly active sites, including cobalt oxide species, nitrogen dopant, and possibly $\mathrm{Co}-\mathrm{N}_{\mathrm{x}}$ species and (2) a 3D conductive, interconnected, hierarchical porous structure for the exposure of active sites and efficient electron and mass transport, the Co-N-GCA exhibited excellent bifunctional catalytic performance and long-term durability in the ORR and OER. These results reveal its promising potential as a low-cost and highly active ORR and OER catalyst for applications in regenerative fuel cells and rechargeable metal-air batteries.

Supplementary Materials: The following are available online at http:/ /www.mdpi.com/2073-4344/8/7/275/s1, Figure S1. HRTEM image of Co-N-GCA. Figure S2: Representative SEM images of N-GCA $(a, b)$ and GCA $(c, d)$ (inset in ( $a$ and c) shows the digital photograph of the corresponding prepared aerogels); Figure S3: XPS survey spectrum of Co-N-GCA and N-GCA (a); high-resolution N 1s spectra of N-GCA (b); Table S1: Surface composition of Co-N-GCA and N-GCA, calculated from XPS results; Table S2: Distribution of each N species, obtained from the fitting results of N1s XPS spectra (normalized to the surface N atoms of each material); Figure S4: CV curves of $20 \mathrm{wt} . \% \mathrm{Pt} / \mathrm{C}$ in $\mathrm{O}_{2}$-saturated $0.1 \mathrm{M} \mathrm{KOH}$ solution; Table S3: Comparison of ORR and OER activity parameters with other recently reported highly active non-noble metal bifunctional electrocatalysts.

Author Contributions: Methodology, X.Q. and Y.L.; Date curation, X.Q. and J.J.; Resources, L.C.; Validation, S.J.; Supervision, H.F.; Writing-original draft, X.Q.; Writing-Review \& Editing, S.L. 
Acknowledgments: The authors would like to acknowledge the financial support of the China Postdoctoral Science Foundation (Project No. 2017M612664), Natural Science Foundation of Guangdong Province (Project No. 2017A030310645).

Conflicts of Interest: The authors declare no conflict of interest.

\section{References}

1. Chu, S.; Majumdar, A. Opportunities and challenges for a sustainable energy future. Nature 2012, 488, 294-303. [CrossRef] [PubMed]

2. Stamenković, V.R.; Fowler, B.; Mun, B.S.; Wang, G.; Ross, P.N.; Lucas, C.A.; Marković, N.M. Improved oxygen reduction activity on $\mathrm{Pt}_{3} \mathrm{Ni}(111)$ via increased surface site availability. Science 2007, 315, $493-497$. [CrossRef] [PubMed]

3. Winter, M.; Brodd, R.J. What are batteries, fuel cells, and supercapacitors? Chem. Rev. 2004, 104, 4245-4270. [CrossRef] [PubMed]

4. McCrory, C.C.L.; Jung, S.; Peters, J.C.; Jaramillo, T.F. Benchmarking Heterogeneous Electrocatalysts for the Oxygen Evolution Reaction. J. Am. Chem. Soc. 2013, 135, 16977-16987. [CrossRef] [PubMed]

5. Chen, Z.; Higgins, D.; Yu, A.; Zhang, L.; Zhang, J. a review on non-precious metal electrocatalysts for PEM fuel cells. Energy Environ. Sci. 2011, 4, 3167-3192. [CrossRef]

6. Tahir, M.; Pan, L.; Idrees, F.; Zhang, X.W.; Wang, L.; Zou, J.J.; Wang, Z.L. Electrocatalytic oxygen evolution reaction for energy conversion and storage: a comprehensive review. Nano Energy 2017, 37, 136-157. [CrossRef]

7. Yeo, B.S.; Bell, A.T. Enhanced Activity of Gold-Supported Cobalt Oxide for the Electrochemical Evolution of Oxygen. J. Am. Chem. Soc. 2011, 133, 5587-5593. [CrossRef] [PubMed]

8. Duan, Z.; Wang, G. a first principles study of oxygen reduction reaction on a $\mathrm{Pt}(111)$ surface modified by a subsurface transition metal $\mathrm{M}(\mathrm{M}=\mathrm{Ni}, \mathrm{Co}$, or Fe). Phys. Chem. Chem. Phys. 2011, 13, 20178-20187. [CrossRef] [PubMed]

9. Zhao, Y.; Kamiya, K.; Hashimoto, K.; Nakanishi, S. Efficient Bifunctional Fe/C/N Electrocatalysts for Oxygen Reduction and Evolution Reaction. J. Phys. Chem. C 2015, 119, 2583-2588. [CrossRef]

10. Zhao, Y.; Chen, S.; Sun, B.; Su, D.; Huang, X.; Liu, H.; Yan, Y.; Sun, K.; Wang, G. Graphene-Co $\mathrm{CO}_{3} \mathrm{O}_{4}$ nanocomposite as electrocatalyst with high performance for oxygen evolution reaction. Sci. Rep. 2015, 5, 7629. [CrossRef] [PubMed]

11. Liang, Y.; Li, Y.; Wang, H.; Zhou, J.; Wang, J.; Regier, T.; Dai, $\mathrm{H} \mathrm{Co}_{3} \mathrm{O}_{4}$ nanocrystals on graphene as a synergistic catalyst for oxygen reduction reaction. Nat. Mater. 2011, 10, 780-786. [CrossRef] [PubMed]

12. Zhang, Z.; Chen, Y.; Bao, J.; Xie, Z.; Wei, J.; Zhou, Z. $\mathrm{Co}_{3} \mathrm{O}_{4}$ Hollow Nanoparticles and Co Organic Complexes Highly Dispersed on N-Doped Graphene: An Efficient Cathode Catalyst for $\mathrm{Li}^{-\mathrm{O}_{2}}$ Batteries. Part. Part. Syst. Charact. 2015, 32, 680-685. [CrossRef]

13. Cao, X.; Zheng, X.; Tian, J.; Jin, C.; Ke, K.; Yang, R. Cobalt Sulfide Embedded in Porous Nitrogen-doped Carbon as a Bifunctional Electrocatalyst for Oxygen Reduction and Evolution Reactions. Electrochim. Acta 2016, 191, 776-783. [CrossRef]

14. Chen, B.; Li, R.; Ma, G.; Gou, X.; Zhu, Y.; Xia, Y. Cobalt sulfide/N,S codoped porous carbon core-shell nanocomposites as superior bifunctional electrocatalysts for oxygen reduction and evolution reactions. Nanoscale 2015, 7, 20674-20684. [CrossRef] [PubMed]

15. Liu, Q.; Jin, J.; Zhang, J. $\mathrm{NiCo}_{2} \mathrm{~S}_{4} @$ graphene as a bifunctional electrocatalyst for oxygen reduction and evolution reactions. ACS Appl Mater. Interfaces 2013, 5, 5002-5008. [CrossRef] [PubMed]

16. Zhang, J.; Qu, L.; Shi, G.; Liu, J.; Chen, J.; Dai, L. N,P-Codoped Carbon Networks as Efficient Metal-free Bifunctional Catalysts for Oxygen Reduction and Hydrogen Evolution Reactions. Angew. Chem. Int. Ed. Engl. 2016, 55, 2230-2234. [CrossRef] [PubMed]

17. Qu, K.G.; Zheng, Y.; Dai, S.; Qiao, S.Z. Graphene oxide-polydopamine derived N, S-codoped carbon nanosheets as superior bifunctional electrocatalysts for oxygen reduction and evolution. Nano Energy 2016, 19, 373-381. [CrossRef]

18. Qiao, X.; Liao, S.; Zheng, R.; Deng, Y.; Song, H.; Du, L. Cobalt and Nitrogen Codoped Graphene with Inserted Carbon Nanospheres as an Efficient Bifunctional Electrocatalyst for Oxygen Reduction and Evolution. ACS Sustain. Chem. Eng. 2016, 4, 4131-4136. [CrossRef] 
19. Zhang, J.; Zhao, Z.; Xia, Z.; Dai, L. a metal-free bifunctional electrocatalyst for oxygen reduction and oxygen evolution reactions. Nat. Nanotechnol. 2015, 10, 444-452. [CrossRef] [PubMed]

20. Petrie, J.R.; Cooper, V.R.; Freeland, J.W.; Meyer, T.L.; Zhang, Z.; Lutterman, D.A.; Lee, H.N. Enhanced Bifunctional Oxygen Catalysis in Strained $\mathrm{LaNiO}_{3}$ Perovskites. J. Am. Chem. Soc. 2016, 138, $2488-2491$. [CrossRef] [PubMed]

21. He, D.; Xiong, Y.; Yang, J.; Chen, X.; Deng, Z.; Pan, M.; Li, Y.; Mu, S. Nanocarbon-intercalated and Fe-N-codoped graphene as a highly active noble-metal-free bifunctional electrocatalyst for oxygen reduction and evolution. J. Mater. Chem. A 2017, 5, 1930-1934. [CrossRef]

22. Su, Y.; Zhu, Y.; Jiang, H.; Shen, J.; Yang, X.; Zou, W.; Chen, J.; Li, C. Cobalt nanoparticles embedded in $\mathrm{N}$-doped carbon as an efficient bifunctional electrocatalyst for oxygen reduction and evolution reactions. Nanoscale 2014, 6, 15080-15089. [CrossRef] [PubMed]

23. Wu, Y.; Zang, J.; Dong, L.; Zhang, Y.; Wang, Y. High performance and bifunctional cobalt-embedded nitrogen doped carbon/nanodiamond electrocatalysts for oxygen reduction and oxygen evolution reactions in alkaline media. J. Power Sources 2016, 305, 64-71. [CrossRef]

24. Qiao, X.; You, C.; Shu, T.; Fu, Z.; Zheng, R.; Zeng, X.; Li, X.; Liao, S. a one-pot method to synthesize high performance multielement co-doped reduced graphene oxide catalysts for oxygen reduction. Electrochem. Commun. 2014, 47, 49-53. [CrossRef]

25. Liu, X.; Amiinu, I.S.; Liu, S.; Cheng, K.; Mu, S. Transition Metal/Nitrogen dual-doped Mesoporous Graphene-like Carbon Nanosheets for Oxygen Reduction and Evolution Reactions. Nanoscale 2016, 8, 13311-13320. [CrossRef] [PubMed]

26. Mao, S.; Wen, Z.; Huang, T.; Hou, Y.; Chen, J. High-performance bi-functional electrocatalysts of 3D crumpled graphene-cobalt oxide nanohybrids for oxygen reduction and evolution reactions. Energy Environ. Sci. 2014, 7, 609-616. [CrossRef]

27. Yasuda, S.; Furuya, A.; Uchibori, Y.; Kim, J.; Murakoshi, K. Iron-Nitrogen-Doped Vertically Aligned Carbon Nanotube Electrocatalyst for the Oxygen Reduction Reaction. Adv. Funct. Mater. 2016, 26, 738-744. [CrossRef]

28. Zhao, J.; Liu, Y.; Quan, X.; Chen, S.; Zhao, H.; Yu, H. Nitrogen and sulfur co-doped graphene/carbon nanotube as metal-free electrocatalyst for oxygen evolution reaction: The enhanced performance by sulfur doping. Electrochim. Acta 2016, 204, 169-175. [CrossRef]

29. Tang, C.; Zhang, Q.; Zhao, M.Q.; Huang, J.Q.; Cheng, X.B.; Tian, G.L.; Peng, H.J.; Wei, F. Nitrogen-doped aligned carbon nanotube/graphene sandwiches: Facile catalytic growth on bifunctional natural catalysts and their applications as scaffolds for high-rate lithium-sulfur batteries. Adv. Mater. 2014, 26, 6100-6105. [CrossRef] [PubMed]

30. Zhu, C.; Fu, S.; Song, J.; Shi, Q.; Su, D.; Engelhard, M.H.; Li, X.; Xiao, D.; Li, D.; Estevez, L.; et al. Self-Assembled Fe-N-Doped Carbon Nanotube Aerogels with Single-Atom Catalyst Feature as High-Efficiency Oxygen Reduction Electrocatalysts. Small 2017, 13, 1603407. [CrossRef] [PubMed]

31. Sun, T.T.; Xu, L.B.; Li, S.Y.; Chai, W.X.; Huang, Y.; Yan, Y.S.; Chen, J.F. Cobalt-nitrogen-doped ordered macro-/mesoporous carbon for highly efficient oxygen reduction reaction. Appl. Catal. B Environ. 2016, 193, 1-8. [CrossRef]

32. Wohlgemuth, S.-A.; White, R.J.; Willinger, M.-G.; Titirici, M.-M.; Antonietti, M. a one-pot hydrothermal synthesis of sulfur and nitrogen doped carbon aerogels with enhanced electrocatalytic activity in the oxygen reduction reaction. Green Chem. 2012, 14, 1515-1523. [CrossRef]

33. Guo, D.; Shibuya, R.; Akiba, C.; Saji, S.; Kondo, T.; Nakamura, J. Active sites of nitrogen-doped carbon materials for oxygen reduction reaction clarified using model catalysts. Science 2016, 351, 361-365. [CrossRef] [PubMed]

34. Cui, X.Y.; Yang, S.B.; Yan, X.X.; Leng, J.G.; Shuang, S.; Ajayan, P.M.; Zhang, Z.J. Pyridinic-Nitrogen-Dominated Graphene Aerogels with Fe-N-C Coordination for Highly Efficient Oxygen Reduction Reaction. Adv. Funct. Mater. 2016, 26, 5708-5717. [CrossRef]

35. Liu, X.; Zhou, W.; Yang, L.; Li, L.; Zhang, Z.; Ke, Y.; Chen, S. Nitrogen and sulfur co-doped porous carbon derived from human hair as highly efficient metal-free electrocatalysts for hydrogen evolution reactions. J. Mater. Chem. A 2015, 3, 8840-8846. [CrossRef]

36. Ferrari, A.C.; Robertson, J. Raman spectroscopy of amorphous, nanostructured, diamond-like carbon, and nanodiamond. Philos. Trans. R. Soc. Lond. a Math. Phys. Eng. Sci. 2004, 362, 2477-2512. [CrossRef] [PubMed] 
37. Prabu, M.; Ramakrishnan, P.; Shanmugam, S. CoMn $\mathrm{O}_{4}$ nanoparticles anchored on nitrogen-doped graphene nanosheets as bifunctional electrocatalyst for rechargeable zinc-air battery. Electrochem. Commun. 2014, 41, 59-63. [CrossRef]

38. Wang, H.; Yang, Y.; Liang, Y.; Cui, L.F.; Sanchez Casalongue, H.; Li, Y.; Hong, G.; Cui, Y.; Dai, H. $\mathrm{LiMn}_{1-\mathrm{x}} \mathrm{FexPO}_{4}$ Nanorods Grown on Graphene Sheets for Ultrahigh-Rate-Performance Lithium Ion Batteries. Angew. Chem. 2011, 123, 7502-7506. [CrossRef] 